\title{
A SURVEY OF THE Anopheles MOSQUITOES (DIPTERA: CULICIDAE) ALONG THE ACRE AND PURUS RIVERS, AMAZON BASIN, BRAZIL
}

\author{
Robert H. ZIMMERMAN ${ }^{1}$, Francisco Xavier PAULINO ${ }^{2}$, Mercia de ARRUDA ${ }^{3}$
}

\begin{abstract}
From January 19 to February 25, 1997 an entomological survey of the seringais and larger towns along the Acre and Purus rivers was made as part of the project "Revisitando a Amazônia de Carlos Chagas; da Borracha a Biodiversidade". Eleven anopheline species and 1285 specimens were collected landing on human baits. The four most abundant species were Anopheles albitarsis s.l. $(\mathrm{n}=778)$, A. darlingi $(\mathrm{n}=359)$, A. rangeli $(\mathrm{n}=69)$ and $A$. oswaldoi $(\mathrm{n}=60)$. A total of 252 larvae were collected of which 10 anopheline species were identified. The most abundant species collected were $A$. albitarsis s.1. $(\mathrm{n}=88)$, A deaneorum $(\mathrm{n}=45)$ and $A$. triannulatus $(\mathrm{n}=40)$, The low numbers of Anopheles collected and the absence of the principal malaria vector $A$. darlingi at the seringais sites suggests that they are not high risk malarious areas. Other Diptera collected were Culex sp., Mansonia titillans, Mansonia pseudotitillans, Psorophora ciliata, Psorophora sp., Coquillettidia (Rhynchotaenia) sp., Simulium amazonicum and S. sanguineum.
\end{abstract}

Keywords: Anopheles, Malaria, Amazon

Levantamento dos Mosquitos Anopheles (Diptera: Culiicidae) ao Longo dos Rios Acre e Purus, na Bacia Amazonica, Brasil.

RESUMO - Entre os meses de Janeiro/Fevereiro de 1997, foi realizado um levantamento entomológico nos seringais e comunidades localizadas no vale do rios Acre e Purus, como parte do projeto "Revisitando a Amazônia de Carlos Chagas; da Borracha a Biodiversidade". Foram capturados no processo de pouso, um total de 1.285 anofelinos pertencentes a 11 espécies. As quatro espécies encontradas em maior número foram: Anopheles albitarsis s.1. $(\mathrm{n}=778), A$. darlingi $(\mathrm{n}=359)$, A. rangeli $(\mathrm{n}=69)$ e A. oswaldoi $(\mathrm{n}=60)$. Também foram coletadas um total de 252 larvas pertencentes a 10 espécies de anofelinos. As espécies mais abundantes foram: A. albitarsis $(n=88)$, A, deaneorum $(n=45)$ e A. triannulatus $(n=40)$. O número reduzido de anofelinos coletados e a ausência do $A$. darlingi nos seringais sugere que essa região não é de alta endemicidade para malaria. Outros mosquitos do gênero Diptera coletados foram: Culex sp., Mansonia titillans, Mansonia pseudotitillans. Psorophora ciliata, Psorophora sp. Coquillettidia (Rhynchotaenia) sp., Simulium amazonicum e S. sanguineum.

Palavras chaves: Anopheles, Malaria, Amazonas

\section{INTRODUCTION}

In 1911 Carlos Chagas visited the seringais (rubber production communities) along the Acre and Purus rivers in western Amazon (Fig. 1) (Cruz, 1913). The intent of the journey was to survey the health situation in the local commu- nities. One of the most prevalent diseases that he found was malaria. At the same time, he collected several species of mosquitoes including the suspected malaria vector Cellia albipes $(=A$. (Nys.) albimanus). Since that time very little information has been collected from the riverine communities in the Purus River

1 Pan American Health Organization, Pan American Sanitary Bureau, Regional Office of the World Health Organization, Caixa Postal 08729, 70912-970, Brasilia DF, BRAZIL.

2 Laboratório de Entomologia, Fundação Nacional de Saúde, Coordenação Regional do Acre, Rio Branco AC, BRAZIL.

3 Departmento de Imunologia, Instituto de Pesquisas Aggeu Magalhães, FIOCRUZ, Av. Moraes Rego S/N Campus de UFPE, 50670-420, Recife PE, BRAZIL. 


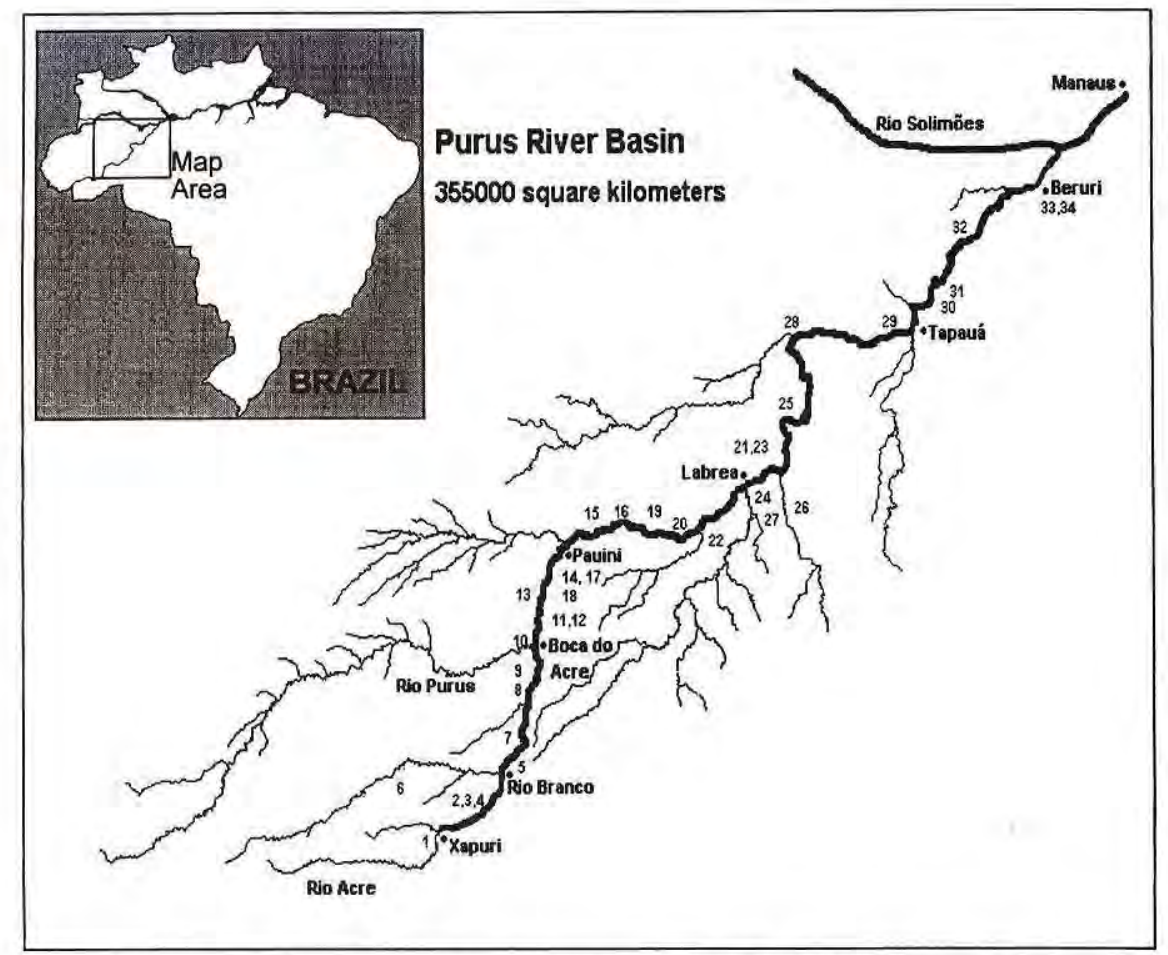

Figure 1. Sites of collection along the rivers Acre and Purus (Purus River Basin). See Tables 1 and 2 for site descriptions.

Basin (Deane et al., 1948; Roberts et al., 1987; Natal et al., 1992; Barata et al., 1993; Fundação Nacional de Saúde, unpublished data).

From January 19 to February 25, 1997, the project "Revisitando a Amazônia de Carlos Chagas; da Borracha a Biodiversidade" conducted a survey of the seringais visited by Carlos Chagas along the Acre and Purus rivers. This article presents the results of the survey of the anopheline fauna.

\section{MATERIALS AND METHODS}

The rivers of the Purus River Basin meander from the foothills of the Andes in Peru to the Solimões river 100 km west of Manaus, Brazil (Fig. 1). They are turbid, whitewater rivers.
Tributaries of the Acre and Purus rivers are usually clearwater streams and rivers (igarapés). Throughout the basin there are many lakes and flooded igapós (black and clearwater floodplains) and várzeas ( muddy inundations) (Pires \& Prance 1985). The ochre to brownish soils are of fluvial origin. Along the main channel the shoreline varies from vertical cliffs, stable sloping shoreline $\left(45^{\circ}\right)$ to beaches. Most towns and rubber communities visited are situated on cliffs overlooking the river or on sloping shorelines. Beach areas were used primarily for subsistence agriculture.

The survey began in the municipality of Xapuri $\left(10^{\circ} 39^{\prime} \mathrm{S}, 68^{\circ} 30^{\prime} \mathrm{W}\right)$, Acre State and ended in the municipality of Beruri, Amazonas State $\left(03^{\circ} 53^{\prime} \mathrm{S}, 61^{\circ}\right.$ 
$\left.22^{\prime} \mathrm{W}\right)$. The latitude and longitude of each collection site was recorded using a Garmin ${ }^{\mathrm{R}}$ geographical positioning system (GPS) recorder.

Adult mosquitoes were collected landing on human baits from 18:0021:00 h. Mosquitoes were put into small holding cartons and kept dry for future identification. Larval surveys were conducted for a minimum of 2 hours at each site by 2 persons; using standardized larval dippers $(500 \mathrm{ml})$. Larval collections were made at all potential breeding sites that were accessible in and around the seringais and the larger towns. Mosquitoes were identified using the keys of Faran (1980) and Consoli \& Lourenço de Oliveira (1994).

A collection of other Culicidae and Simullidae, was made at various seringais.

\section{RESULTS}

The results of the survey are summarized in Tables 1 and 2. Of the 18 adult collections made, 13 were positive for Anopheles. Overall, 11 different Anopheles species and 1285 specimens were collected landing in humans. The four most abundant species were Anopheles albitarsis s.1. $(\mathrm{n}=778)$ A. darlingi $(\mathrm{n}=359)$, A. rangeli $(\mathrm{n}=69)$ and A. oswaldoi $(\mathrm{n}=60)$. The presence and abundance of each species differed by collection site (Tab. 1). The majority of the anophelines collected were from larger towns such as Lábrea and Boca do Acre. The seringais accounted for only $14 \%$ of the total adults collected.

Larval collections were made in 21 localities of which 18 were positive
(Tab. 2). Not all breeding sites in each location were positive. Ten different anopheline species were collected during the survey and a total of 252 larvae were identified. The most abundant species collected were $A$. albitarsis s.l. $(\mathrm{n}=88), A$. deaneorum $(\mathrm{n}=45)$ and $A$. triannulatus $(\mathrm{n}=40)$. Only one $A$. darlingi larva was collected at Yapui on Rio Ituxi, Lábrea. The main larval breeding sites were river backwaters, streams, and artificial ponds. Várzeas and igapós were consistently negative for Anopheles larvae.

Along with the anophelines, we collected several other genera of mosquitoes. Culex sp. was almost always collected during human biting counts. When one entered the forest in search of larval breeding sites large numbers of Mansonia titillans, Mansonia pseudotitillans, Psorophora ciliata, Psorophora sp., and/or Coquillettidia (Rhynchotaenia) sp. were found biting. Simulium amazonicum and $S$. sanguineum were the daytime biting Simuliidae collected during the survey.

\section{DISCUSSION}

It is difficult to compare the results of this survey with that of Carlos Chagas because the anopheline species collected by Chagas, Cellia albipes Theobald, is a junior synonym of $A$. (Nys.) albimanus whose name was erroneously applied to other anophelines species of the subgenus Nyssorhynchus (Faran, 1980).

The majority of adults and larvae were collected around the larger towns (Tabs. $1 \& 2$ ). The seringais showed very low densities of anophelines. This may have been due to the low 
Table 1. Adult Anopheles collected from the Acre and Purus rivers, Amazon Basin, Brazil, from January 19 to Febuary 22, 1997.

\begin{tabular}{|c|c|c|c|c|c|c|c|c|c|c|c|c|c|}
\hline Municipality & Locality & $\begin{array}{l}\text { Geographic } \\
\text { Coordinates }\end{array}$ & $\begin{array}{l}\text { A. } \\
\text { rangeli }\end{array}$ & $\begin{array}{l}\text { A. } \\
\text { oswaldoi }\end{array}$ & $\begin{array}{l}\text { A. } \\
\text { darlingi }\end{array}$ & $\begin{array}{l}\text { A. } \\
\text { albitarsis s.l. }\end{array}$ & $\begin{array}{l}\text { A. trian- } \\
\text { nulatus }\end{array}$ & $\begin{array}{l}\text { A. } \\
\text { rondoni }\end{array}$ & $\begin{array}{l}\text { A. benar- } \\
\text { rochi }\end{array}$ & $\begin{array}{l}\text { A.brazil- } \\
\text { iensis }\end{array}$ & $\begin{array}{l}\text { A. } \\
\text { minor }\end{array}$ & $\begin{array}{l}\text { A. matto- } \\
\text { grossensis }\end{array}$ & $\begin{array}{l}\text { A. medio- } \\
\text { punctatus }\end{array}$ \\
\hline Xapuri & Larangal' $(1)^{2}$ & $\begin{array}{l}10^{\circ} 39 \mathrm{~S} \\
68^{\circ} 30^{\circ} \mathrm{W}\end{array}$ & 0 & 0 & 0 & 0 & 0 & 0 & 0 & 0 & 0 & 0 & 0 \\
\hline Xapuri & $\begin{array}{l}\text { Sao Francisco } \\
\text { de Iracema' (2) }\end{array}$ & $\begin{array}{l}10^{\circ} 31 ' \mathrm{~S} \\
68^{\circ} 07 \mathrm{~W}\end{array}$ & 0 & 0 & 1 & 1 & 0 & 0 & 0 & 0 & 0 & 0 & 0 \\
\hline Xapuri & Paraiso' $^{\prime}(3)$ & $\begin{array}{l}10^{\circ} 15 \cdot \mathrm{S} \\
67^{\circ} 48^{\prime} \mathrm{W}\end{array}$ & 19 & 34 & 0 & 0 & 3 & 0 & 0 & 0 & 1 & 1 & 1 \\
\hline Rio Branco & $\begin{array}{l}\text { Univ.Federal } \\
\text { Acre (5) }\end{array}$ & - & 0 & 2 & 0 & 0 & 0 & 0 & 0 & 0 & 0 & 0 & 0 \\
\hline Rio Branco & $\begin{array}{l}\text { Novo Horizonte' } \\
\text { (6) }\end{array}$ & $\begin{array}{l}09^{\circ} 55^{\circ} \mathrm{S} \\
67^{\circ} 37^{\prime} \mathrm{W}\end{array}$ & 0 & 0 & 0 & 0 & 0 & 0 & 0 & 0 & 0 & 0 & 0 \\
\hline Rio Branco & $\begin{array}{l}\text { Bela Vista' (7) } \\
\text { Sao Francisco' }\end{array}$ & " " & 0 & 0 & 0 & 0 & 0 & 0 & 0 & 0 & 0 & 0 & 0 \\
\hline $\begin{array}{l}\text { Boca do } \\
\text { Acre }\end{array}$ & $\begin{array}{l}\text { Sao Francisco' } \\
\text { (8) }\end{array}$ & - & 1 & 2 & 0 & 0 & 0 & 0 & 0 & & 0 & & 0 \\
\hline Rio Branco & $\begin{array}{l}\text { Riozinho do } \\
\text { Rola' (9) }\end{array}$ & $\begin{array}{l}10^{0} 03 ' \mathrm{~S} \\
67^{\circ} 51 \mathrm{w}\end{array}$ & 48 & 19 & 0 & 17 & 4 & 2 & 2 & 0 & 0 & 0 & 0 \\
\hline $\begin{array}{l}\text { Boca do } \\
\text { Acre }\end{array}$ & $\begin{array}{l}\text { Bairro } \\
\text { Macacheral (10) }\end{array}$ & $\begin{array}{l}08^{\circ} 45^{\prime S} \mathrm{~S} \\
67^{\circ} 23^{\prime} \mathrm{W}\end{array}$ & 1 & 1 & 0 & 496 & 0 & 0 & 3 & 0 & 0 & 0 & 0 \\
\hline $\begin{array}{l}\text { Boca do } \\
\text { Acre }\end{array}$ & $\begin{array}{l}\text { Bairro } \\
\text { Macacheral (11) }\end{array}$ & “ “ & 0 & 0 & 0 & 264 & 0 & 0 & 0 & 0 & 0 & 0 & 0 \\
\hline Pauini & $\begin{array}{l}\text { Bairro Cozoma } \\
\text { (14) }\end{array}$ & $\begin{array}{l}07^{\circ} 43 \mathrm{~S} \\
67^{\circ} 00^{\prime} \mathrm{w}\end{array}$ & 0 & 0 & 33 & 0 & 0 & 0 & 0 & 0 & 0 & 0 & 0 \\
\hline Pauini & 'Guajarrā' (15) & $\begin{array}{l}07^{\circ} 41^{\prime} \mathrm{S} \\
06^{\circ} 53^{\mathrm{W}} \mathrm{W}\end{array}$ & 0 & 0 & 0 & 0 & 0 & 0 & 0 & 0 & 0 & 0 & 0 \\
\hline Pauini & $\begin{array}{l}\text { Sta. Candida' } \\
\text { (16) }\end{array}$ & $\begin{array}{l}07^{\circ} 42^{\prime} \mathrm{S} \\
65^{\circ} 41^{\prime} \mathrm{W}\end{array}$ & 0 & 0 & 0 & 0 & 0 & 0 & 0 & 0 & 0 & 0 & 0 \\
\hline Lábrea & Lábrea (21) & $\begin{array}{l}07^{\circ} 15 \mathrm{~S} \\
64^{\circ} 48^{\prime} \mathrm{w}\end{array}$ & 0 & 0 & 260 & 0 & 0 & 0 & 0 & 2 & 0 & 0 & 0 \\
\hline Camutama & $\begin{array}{l}\text { Bairro Tanque } \\
\text { (25) }\end{array}$ & $\begin{array}{l}06^{\circ} 32^{\prime} \mathrm{S} \\
64^{\circ} 23 \mathrm{w}\end{array}$ & 0 & 0 & 29 & 0 & 0 & 0 & 0 & 0 & 0 & 0 & 0 \\
\hline Lábrea & $\begin{array}{l}\text { Camahá' (26) } \\
64^{\circ} 20^{\circ} \mathrm{W}\end{array}$ & $06^{\circ} 42^{\prime} \mathrm{S}$ & 0 & 0 & 29 & 0 & 0 & 0 & 0 & 0 & 0 & 0 & 0 \\
\hline Tapauá & $\begin{array}{l}\text { Boca do } \\
\text { Tapauá (28) }\end{array}$ & $\begin{array}{l}05^{\circ} 45^{\prime} \mathrm{S} \\
64^{\circ} 23^{\prime} \mathrm{W}\end{array}$ & 0 & 0 & 0 & 0 & 0 & 0 & 0 & 0 & 0 & 0 & 0 \\
\hline Tapauá & $\begin{array}{l}\text { Bairro Fernando } \\
\text { Mendes (30) }\end{array}$ & $\begin{array}{l}05^{\circ} 37 \mathrm{~s} \\
63^{\circ} 11 \mathrm{w}\end{array}$ & 0 & 2 & 7 & 0 & 0 & 0 & 0 & 0 & 0 & 0 & 0 \\
\hline Total & & & 69 & 60 & 359 & 778 & 7 & 2 & 5 & 2 & 1 & 1 & 1 \\
\hline
\end{tabular}

2. 'Seringais; all other localities are villages along the rivers.

2 () Locality number from Figure 1. 


\begin{tabular}{|c|c|c|c|c|c|c|c|c|c|c|c|c|}
\hline Municipality & Locality & $\begin{array}{l}\text { Geographic } \\
\text { Coordinates }\end{array}$ & $\begin{array}{l}\text { A. nunez- } \\
\text { tovari }\end{array}$ & $\begin{array}{c}\text { A. } \\
\text { rangeli }\end{array}$ & $\begin{array}{c}\text { A. } \\
\text { oswaldoi }\end{array}$ & $\begin{array}{c}\text { A. } \\
\text { darlingi }\end{array}$ & $\begin{array}{l}\text { A. albi- } \\
\text { tarsis s.I. }\end{array}$ & $\begin{array}{l}\text { A. trian- } \\
\text { nulatus }\end{array}$ & $\begin{array}{c}\text { A. } \\
\text { shanoni }\end{array}$ & $\begin{array}{c}\text { A. brazil- } \\
\text { lensis }\end{array}$ & $\begin{array}{l}\text { A. deane- } \\
\text { orum }\end{array}$ & $\begin{array}{l}\text { A. per- } \\
\text { yassui }\end{array}$ \\
\hline Xapuri & Larangal $^{2}(1)$ & $\begin{array}{l}10^{\circ} 39^{\prime} \mathrm{S} \\
68^{\circ} 30^{\prime} \mathrm{W}\end{array}$ & 0 & 0 & 0 & 0 & 2 & 6 & 0 & 0 & 0 & 0 \\
\hline Xapuri & $\begin{array}{l}\text { Sao Fransisco } \\
\text { de Iracema }\end{array}$ & $\begin{array}{l}10^{\circ} 31 \mathrm{~S} \\
68^{\circ} 07 \mathrm{~W}\end{array}$ & 0 & 0 & 0 & 0 & 0 & 13 & 0 & 0 & 0 & 0 \\
\hline $\begin{array}{l}\text { Xapuri } \\
\text { Boca do Acre }\end{array}$ & $\begin{array}{l}\text { Itúv }(4) \\
\text { Bairro Praia }\end{array}$ & $\overline{08^{\circ}} 45 \mathrm{~S}$ & 0 & 0 & 0 & 0 & 8 & 0 & 0 & 0 & 0 & 0 \\
\hline Boca do Acre & $\begin{array}{l}\text { do Gado (12) } \\
\text { Rio Mapiáz (13) }\end{array}$ & $67^{\circ} 23^{W}$ & $\begin{array}{l}0 \\
1\end{array}$ & $\begin{array}{l}1 \\
2\end{array}$ & $\begin{array}{l}0 \\
0\end{array}$ & $\begin{array}{l}0 \\
0\end{array}$ & $\begin{array}{c}53 \\
2\end{array}$ & $\begin{array}{l}0 \\
0\end{array}$ & $\begin{array}{l}0 \\
0\end{array}$ & $\begin{array}{l}0 \\
0\end{array}$ & $\begin{array}{l}31 \\
8\end{array}$ & $\begin{array}{l}0 \\
0\end{array}$ \\
\hline Pauini & Cidade Alta (17) & $\begin{array}{l}07^{\circ} 43^{\prime S} \mathrm{~S} \\
67^{\circ} 00^{\circ} \mathrm{W}\end{array}$ & 0 & 0 & 0 & 0 & 4 & 0 & 0 & 0 & 0 & 0 \\
\hline $\begin{array}{l}\text { Pauini } \\
\text { Pauini }\end{array}$ & $\begin{array}{l}\text { Bairro Cozama (18) } \\
\text { Cachoeira do }\end{array}$ & $07^{\circ} 40^{\prime \prime} \mathrm{s}$ & 0 & 0 & 0 & 0 & 0 & 0 & 1 & 0 & 0 & 0 \\
\hline Pauini & Hilario $^{2}(19)$ & $65^{\circ} 59 \mathrm{~W}$ & 0 & 0 & 0 & 0 & 0 & 0 & 0 & 0 & 0 & 0 \\
\hline Pauini & Santa Candida ${ }^{2}(20)$ & $\begin{array}{l}77^{\circ} 42 \mathrm{~S} \\
65^{\circ} 41^{\prime} \mathrm{W}\end{array}$ & 0 & 0 & 0 & 0 & 0 & 0 & 0 & 0 & 0 & 0 \\
\hline Lábrea & Marraăa $^{2}(22)$ & $\begin{array}{l}07^{\circ} 28^{\prime} \mathrm{S} \\
65^{\circ} 18^{\prime} \mathrm{W}\end{array}$ & 0 & 0 & 0 & 0 & 1 & 0 & 0 & 0 & 5 & 0 \\
\hline Lábrea & Faz. Paiva (23) & $\begin{array}{l}07^{\circ} 15^{\mathrm{S} S} \\
64^{\circ} 48^{\mathrm{W}} \mathrm{W}\end{array}$ & 0 & 0 & 0 & 0 & 0 & 15 & 0 & 3 & 0 & 0 \\
\hline $\begin{array}{l}\text { Lábrea } \\
\text { Lábrea }\end{array}$ & $\begin{array}{l}\text { B.R. } 230, \mathrm{Km} \mathrm{3} 3(24) \\
\text { Rio ituxi, Yapui } \\
\text { (27) }\end{array}$ & $\begin{array}{l}07 \% " \\
07^{\circ} 19 . \mathrm{S} \\
64^{\circ} 55 \mathrm{~W}\end{array}$ & $\begin{array}{l}0 \\
3\end{array}$ & $\begin{array}{l}0 \\
0\end{array}$ & $\begin{array}{l}0 \\
0\end{array}$ & $\begin{array}{l}0 \\
1\end{array}$ & $\begin{array}{c}12 \\
0\end{array}$ & $\begin{array}{l}6 \\
0\end{array}$ & $\begin{array}{l}0 \\
0\end{array}$ & $\begin{array}{l}8 \\
0\end{array}$ & $\begin{array}{l}1 \\
0\end{array}$ & : \\
\hline Lábrea & Camahá $^{2}(26)$ & $\begin{array}{l}06^{\circ} 42^{\prime S} \mathrm{~S} \\
64200^{\circ} \mathrm{W}\end{array}$ & 0 & 0 & 0 & 0 & 0 & 0 & 0 & 0 & 0 & 0 \\
\hline Tapauá & Boca de Tapauá (28) & $\begin{array}{l}05^{\circ} 45^{\circ} \mathrm{S} \\
64^{\circ} 23^{\mathrm{W}} \mathrm{W}\end{array}$ & 0 & 0 & 0 & 0 & 4 & 0 & 0 & 0 & 0 & 0 \\
\hline Tapauá & $\begin{array}{l}\text { Vila Tapauã }{ }^{2} \\
\text { (Floresta) (29) }\end{array}$ & $\begin{array}{l}05^{\circ} 42^{\circ} \mathrm{S} \\
64^{\circ} 24^{2} \mathrm{~W}\end{array}$ & 0 & 0 & 0 & 0 & 0 & 0 & 0 & 0 & 0 & 2 \\
\hline Tapauá & Bairro Açais (30) & $\begin{array}{l}05^{\circ} 37 \mathrm{~S} \\
63^{\circ} 11 \mathrm{~W}\end{array}$ & 0 & 0 & 0 & 0 & 2 & 0 & 0 & 0 & 0 & 0 \\
\hline Tapauá & Tambaquil ${ }^{2}(31)$ & $\begin{array}{l}05^{\circ} 11 \mathrm{~S} \\
62^{\circ} 54^{\mathrm{W}} \mathrm{W}\end{array}$ & 0 & 0 & 0 & 0 & 0 & 0 & 0 & 0 & 0 & 2 \\
\hline Tapauá & Aracari' (32) & $\begin{array}{l}05^{\circ} 11^{\prime S} \\
62^{\circ} 54^{\prime} \mathrm{W}\end{array}$ & 0 & 0 & 15 & 0 & 0 & 0 & 0 & 0 & 0 & 0 \\
\hline Beruri & $\begin{array}{l}\text { Nova Trombeta } \\
\text { (33) }\end{array}$ & $\begin{array}{l}04^{\circ} 28^{\prime} \mathrm{S} \\
81^{\circ} 22^{\prime} \mathrm{W}\end{array}$ & 0 & 0 & 0 & 0 & 0 & 0 & 0 & 0 & 0 & 6 \\
\hline Beruri & Boca do Assair (34) & $\begin{array}{l}03^{\circ} 53^{\prime S} \\
81^{\circ} 22^{\prime} \mathrm{W}\end{array}$ & 0 & 0 & 8 & 0 & 0 & 0 & 0 & 0 & 0 & 0 \\
\hline & TOTAL & & 4 & 3 & 23 & 1 & 88 & 40 & 1 & 11 & 45 & 10 \\
\hline
\end{tabular}

\footnotetext{
Thirty Anopheles larvae could not be identified to species.

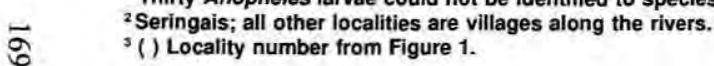


number of positive breeding sites. It was somewhat surprising that the flooded várzea and igapós were negative because they have been considered important anopheline breeding sites; particularly for $A$. darlingi (Forattini, 1962). It may be due to the fact that the Acre and Purus rivers are muddy water rivers and $A$. darling $i$ prefers clear water (Deane et al., 1948). The presence of adult $A$. darlingi next to clear water igarapés in the towns of Lábrea and Canutama and along the Mucuim river would support this conclusion.

The four most abundant Anopheles collected in this study, A. albitarsis s.1., A. darlingi, $A$. rangeli and $A$. oswaldoi, have been implicated in the transmission of malaria in the Amazon Basin (Arruda et al., 1986; Deane, 1986; Zimmerman, 1992; Branquinho et al., 1993). A. albitarsis is a species complex (Wilkerson et al., 1995) and only one other member of this complex, $A$. deaneorum, can be separated by morphological characteristics (Rosa-Freitas, 1989). In this survey we did not collect any adults of $A$. deaneorum (Tab. 2).

The results of our survey complement previous studies on Anopheles from Acre State (Deane et al., 1948; Natal et al., 1992; Barata et al., 1993) and Amazonas State (Deane et al., 1948; Xavier \& Mattos, 1976; Roberts et al. 1987; Tadei et al., 1993; Osorio Quintero et al., 1996; Baggio, unpublished data). Deane et al. (1948) surveyed several sites from Acre State to Para State. During their survey anophelines were collected from Xapuri, Rio Branco, Boca do Acre, and Lábrea. The results of our survey at these same sites were similar to Deane et al. (1948). The major diference was that we could separate $A n$. deaneorum from An. albitarsis s.l. and they could not. In conclusion, we extend the distribution records for several species of anophelines, including the new species $A n$. deaneorum, to include previously unsurveyed stretches of the Acre and Purus rivers from the municipality of Xapuri, Acre State, to Beruri, Amazonas State.

The low number of anophelines collected at the seringais sites suggests that the seringais along the Acre and Purus rivers are not high-risk malarious areas. This is supported by the results of the malaria serology survey conducted during this study (Arruda, unpublished data). However, Deane et al. (1948) found seringais along Rio Zinho, Acre State to have high malaria prevalence and large numbers of $A$. darlingi. In contrast, our entomological data suggest that the risk of malaria transmission in the communities along and next to the igarapés is higher. Further research is warranted to confirm this conclusion.

\section{ACKNOWLEDGEMENTS}

To Casa Oswaldo Cruz/ FIOCRUZ for organizing the survey and partial funding. To the Panamerican Health Organization, Pan American Sanitary Bureau, Regional Office of the World Health Organization for partial funding. Fundação Nacional de Saúde, Coordenação Regional do Acre provided logistic and entomological support. Drs. G. Calderón and R. Galvão assisted with the identification of the Diptera.

\section{Literature cited}

Arruda, M.; Carvalho, M.B.; Nussenzweig, R.S.; Maracic, M.; Ferreira, A.W.; Cochrane, A.H. 1986. Potential vectors of malaria and their different susceptibility to Plasmo- 
dium falciparum and Plasmodium vivax in northern Brazil identified by immunoassay. Am. J. Trop. Med. Hyg., 35: 873-881.

Barata, J.M.S.; Natal, D; Lagos, C.B.T.; Urbinatti, P.R.; Rocha R.M. 1993. Fauna de mosquitos (Diptera:Culicidae) da bacia do rio Purus (Acre, Brasil). Identificação de formas imaturas em área de projeto de colonização. Revta bras. Ent. 37: 365-368.

Branquinho, M.S.; Taipe Lagos, C.B.T.; Rocha, R.M.; Natal, D.; Barata, J.M.; Cochrane, A.H.; Nardin, E.; Nussenzweig, R.S.; Kloetzel, J.K. 1993. Anophelines in the state of Acre, Brazil, infected with Plasmodium falciparum, $P$. vivax, the $P$. vivax variant $P$. vivax VK247 and P. malariae. Trans. R. Soc. Trop. Med. Hyg. 87: 391-394.

Consoli, R.A.G.B.; Lourenço De Oliveira, R. 1994. Principais mosquitos de importância sanitária no Brasil. Editora Fiocruz, Rio de Janeiro, 225pp.

Cruz, O. 1913. Relatório sobre as condições medico-sanitárias do vale do Amazonas, Typographia do Journal do Commercio, Rio de Janeiro.

Deane, L. M. 1986. Malaria vectors in Brazil. Mem. Inst. Oswaldo Cruz, 81 (suppl.3): 5-14.

Deane, L.M.; Causey, R.; Deane, M.P. 1948. Notas sôbre a distribuição e a biologia dos anofelinos das regiões nordestina e Amazônica do Brasil. Rev. Serv. Espec. Saúde Públ., 1: 827-965.

Faran, M.E. 1980. Mosquito studies (Diptera, Culicidae). XXXIV. A revision of the Albimanus Section of the subgenus Nyssorhynchus of Anopheles. Contrib. Am. Entomol. Inst. (Ann Arbor) 15: 1-215.

Forattini, O.P. 1962. Entomologia Médica. I. Parte Geral, Diptera, Anophelini. Fac. Hig. Saúde Publ., São Paulo, 662pp.

Natal, D.; Barata, J.M.S.; Lagos, C.B.T.; Rocha, R.M. 1992. Nota sobre culicideos (Diptera: Culicidae) da bacia do rio Purus, Acre Amazònia (Brasil). Rev. Saúde públ. São Paulo, 26: 129-131.
Osorio Quintero, L.; Dutary Thatcher, B.; Tadei, P. 1996. Biologia de anofelinos Amazônicos, XXI. Ocorrência de espécies de Anopheles e outros culicideos na área de influência da hidrelétrica de Balbina - cinco anos após o enchimento do reservatório. Acta Amazonica, 26: 281-296.

Pires, J.M.; Prance, G.T. 1985. The vegetation types of the Brazilian Amazon, pp. 109B145. In: Prance, G.T.; Lovejoy, T.E. (Eds). Key environments, Amazônia. Pergamon, New York.

Roberts, D.R.; Alecrim, W.D.; Tavares, A.M.; Radke, M.G. 1987. The house-frequenting, host-seeking and resting behavior of Anopheles darlingi in southeastern Amazonas, Brazil. J. Amer: Mosq. Control Assoc., 3: 433-441.

Rosa-Freitas, M.G. 1989. Anopheles (Nyssorhynchus) deaneorum; a new species in the albitarsis complex (Diptera: Culicidae). Mem. Inst. Oswaldo Cruz, 84: 535-43.

Tadei, W.P.; Santos, J.M.M.; Scarpassa, V.M.; Rodrigues, I.B. 1993. Incidência, distribuição, e aspectos ecológicos de espécies de Anopheles (Diptera: Culicidae), em regiões naturais e sob impacto ambiental da Amazônia Brasileira. In: Ferreira, E.J.G.; Santos, G.M.; Leão, E.L.M.; Oliveira, L.A. (Eds). Bases cientificas para estratégias de preservação e desenvolvimento $d a$ Amazônia. Vol. 2. INPA, Manaus, AM.: 167-196.

Wilkerson, R.C.; Gaffigan, T.V.; Lima, J.B.1995. Identification of species related to Anopheles (Nyssorhynchus) albitarsis by random amplified polymorphic DNA-polymerase chain reaction (Diptera: Culicidae). Mem. Inst. Oswaldo Cruz, 90: 721-732.

Xavier, S.H.; Mattos, S. 1976. Geographical distribution of Culicinae in Brazil - IV. State of Amazonas (Diptera, Culicidae). Mosq. Syst., 8: 386-412.

Zimmerman, R.H. 1992. Ecology of malaria vectors in the Americas and future direction. Mem. Inst. Oswaldo Cruz, 87, (suppl 3): $371-383$. 